\title{
DESIGN OF A MINIMAL SIGNALING BAS] BANDWIDTH BROKERING PROTOCOL
}

\author{
Mohit Agarwal and Abhik Majumdar \\ Department of Electrical Engineering and Computer Sciences \\ University of California, Berkeley, CA 94720 \\ \{mohit, abhik\}@eecs.berkeley.edu
}

Abstract The DiffServ architecture uses automated agents known as Bandwidth Brokers for provisioning, allocating and managing QoS resources within domains as well as communicating with agents of other domains so that QoS guarantees can be provided across domains. The Bandwidth Brokers attempt to establish an end-to-end Quality of Service through signaling between each other. This leads to an implicit trade-off between scalability and the end- to-end QoS guarantees that can be provided. In this work we propose a new Bandwidth Broker signaling mechanism and show, through extensive simulations, that it is possible to specify endto-end QoS guarantees that are sufficiently high even when signaling is minimal and link utilizations are very high. We have focused solely on the inter-domain communication between the brokers, assuming that the intra-domain issues have been handled separately. A major aspect of our design is that negotiations between Bandwidth Brokers are modeled as a function of available bandwidth and cost to the respective ISPs of the domains (whom the Bandwidth Brokers represent in business dealings). This makes our approach close to the real world scenario in which brokers will always try to end up with a profit after each negotiation process.

Keywords: Bandwidth Broker, Quality of Service, DiffServ

\section{INTRODUCTION}

Internet applications designers are increasingly feeling the need for guarantees in network performance. Moreover, the nature of these service guarantees varies with the applications. For example, applications such as voice-over-IP and video-conferencing require low round trip delays as well as a small jitter while in audio streaming larger round-trip delays are acceptable if jitter is low. Generally, a best effort kind of service may not be good enough to support such applications.

The original version of this chapter was revised: The copyright line was incorrect. This has been corrected. The Erratum to this chapter is available at DOI: 10.1007/978-0-387-35584-9_19 
IETF standards for Integrated Services (Intserv) in routers [1] and the end-to-end reservation set-up protocol (RSVP) [2] were proposed to provide such service guarantees in the Internet. These standards introduce a per-flow state in network nodes in the network nodes along the path of the flow. While fine-grain service agreements can be supported through these approaches, these models do not scale well since maintaining per flow state for thousands of flows incredibly increases the complexity of the routers. The resource requirements (processing and storage) for running RSVP on a router increase proportionally with the number of separate sessions (i.e., RSVP reservations). Hence, supporting numerous small reservations on a high-bandwidth link may easily over tax the routers [8].

A more scalable approach called Differentiated Services (DiffServ) was proposed in [3]. Scalability is achieved here, by introducing a notion of "flow aggregation" whereby all flows at a network node belonging to a particular "class" are treated as a combined flow. Classification, marking, shaping and policing operations are performed only at the network boundaries or hosts. Typically, this is thought of as a two-tier model where several autonomous systems exist and each one of them is responsible for internally managing traffic to provide class-based services while they communicate with peer autonomous systems to set up service agreements that ensure that such services can be offered on an interdomain or inter-network basis.

The DiffServ architecture defines the Service Level Agreement or SLA as a service contract between a customer and a provider that specifies the forwarding service a customer should receive. A customer may be a user organization (source domain) or another Diffserv domain. Diffserv architecture uses automated agents known as Bandwidth Brokers for negotiating SLAs between different autonomous systems and managing resources within the domain. In this paper, we assume that mechanisms for resource management within the domain exist and propose a signaling mechanism for setting up inter domain service agreements.

There has been a substantial amount of prior work in the area of implementation of Bandwidth Brokers. The Internet2 Qbone BB Advisory Council's paper on Bandwidth Broker requirements for Internet2 Qbone Deployment [4] discusses the requirements of a Bandwidth Broker. It provides certain guidelines for implementing Bandwidth Brokers within a DiffServ framework and proposes various deployment phases designed to provide a staged approach to providing inter-domain communication. Both inter - and intra - domain issues are discussed.

A 'two-tier resource management model for the Internet' is suggested in [5]. In the realization of this model a Bandwidth Broker acts as a 
resource manager for each administrative domain and neighboring brokers communicate with each other to establish inter-domain resource agreements.

An agent based architecture for quantitative service provisioning in DiffServ capable networks is proposed in [6]. The Bandwidth Broker is the agent responsible for admission control. The architecture provides for resource reservations for aggregated virtual leased lines between network domains. This approach provides support for both immediate and advance reservations.

From these studies it becomes evident that a critical aspect of the design and deployment of Bandwidth Brokers is the inherent trade-off between the end-to-end Quality of Service (QoS) guarantees that can be provided and the amount of signaling needed. Before a request for differentiated service is granted, the Bandwidth Broker needs to check for the availability of the resources that were requested. This involves analyzing the state of QoS resources within the domain as well as signaling to brokers of neighboring domains. Different signaling protocols are evaluated in [7] and an alternative signaling protocol is proposed.

In this paper, our main contribution is the development of a novel signaling protocol for inter-domain resource reservation. We model the negotiation between two brokers for a SLA as a monetary transaction which is based on the estimate of expected cost for the new SLA. Each broker maintains this estimate for each for its adjacent domains and updates it based on the distribution of its outgoing traffic and a history of previous negotiations. This signaling is very scalable and experimental results on a simulated system show that very good QoS can be achieved even when the resource utilization is very high.

The rest of the paper is organized as follows. In section 2 we present various signaling methods that were previously studied in [7]. In section 3 the proposed signaling mechanism is presented. Experimental results are discussed in section 4 . We conclude the paper with a discussion and suggestion for future work in section 5 .

\section{BANDWIDTH BROKERS AND SIGNALING}

As mentioned above Bandwidth Brokers are responsible for the overall management of the QoS resources within their domains. This includes the configuring of the routers within the domain and the edge routers on links to adjacent domains. The Bandwidth Brokers also manage interdomain communication by talking to peer bandwidth brokers in adjacent domains. They gather and monitor the state of QoS resources within 
their domains and on the edges of the domains. The brokers make admission control decisions based on this information, certain intra-domain policy specifications and the information they gather by talking to peer Bandwidth Brokers in adjacent downstream domains. The Bandwidth Brokers are responsible for negotiating and renegotiating SLAs between adjacent domains. The SLAs will specify the aggregate amount of QoS traffic crossing domains. This aggregation is a key feature of DiffServ architecture, which ensures scalability in terms of network size and traffic. The state sharing between brokers is done through a signaling protocol.

As was mentioned above, there is an inherent trade-off between the amount of signaling and the QoS guarantees that can be provided. This signaling may include notifying the adjacent brokers of how much Diffserv traffic is being sent and/or renegotiating the SLA to reserve additional bandwidth. There are two possibilities:

- No notification: The simplest possible signaling protocol is when brokers make admission control decisions based on the state of QoS resources within the domain and the SLAs that exist with adjacent brokers. Naturally, it is almost impossible to provide QoS guarantees in such a scenario. However, the advantages of the protocol are that there are no notification costs and scalability is not a problem. It is possible, though, to provide some end-to-end statistical QoS guarantees by over-provisioning of the SLAs.

- End-to-End notification: Diametrically opposite of the no notification scenario is the end- to-end signaling protocol. Here each broker notifies its neighboring broker(s) of new Diffserv traffic before actually admitting the new request. If necessary, the broker also renegotiates the SLA. The neighboring brokers operate in a similar manner by notifying the downstream brokers. Hence, before a request is accepted all the brokers from the traffic source to the sink have been notified. While such an approach will enable the brokers to give end-to-end QoS guarantees, it is also obvious that such a method is not scalable because of the massive overheads in terms of bandwidth, delays and router complexity.

A "limited notification" signaling protocol was proposed in [7] as an alternative to the no-notification and end-to-end notification approaches. The proposed approach is to de-couple the notification process from the request reservation process. When a Bandwidth Broker receives a notification announcing DiffServ traffic on an incoming link it estimates the impact on the local network and the impact on the outgoing links. It then uses these estimates and a threshold called the reservation threshold to determine whether to renegotiate the SLA. Another threshold called 
the notification threshold is used to determine whether to notify other Bandwidth Brokers. Typically this threshold is lower than the reservation threshold. SLAs are over- provisioned so that better (statistical) QoS guarantees may be provided. To limit the number of notifications, one notification is used for several aggregated flows. Hence, in this approach, the information regarding the destination of the flows cannot be specified in the SLA. To get around this problem, the use of exponential estimation is proposed, where each Bandwidth Broker maintains a matrix that specifies the probability that traffic coming in on link $i$ will leave on link $j$. Periodically the broker updates the probability matrix based on the past history of how the traffic on an incoming link gets distributed into outgoing links.

Our proposed algorithm is, in part, inspired by the viability of the limited notification ideas as shown in [7]. We also use the idea of exponential estimation as suggested in [7]. However, [7] may face a scalability problem when the resource utilization is high and the amount of over provisioning is small. In such a scenario, most re-negotiation notifications would need to propagate from source of the traffic to its sink. In contrast, in our model, notifications/re-negotiations do not propagate beyond the adjacent Bandwidth Brokers and hence scalability is never a problem. Further, as explained below, we integrate a design for the monetary transactions involved in the negotiation process into our model. In fact, in our algorithm, the re-negotiations between brokers are based on comparisons between the expected revenue to a broker and the cost it incurs for renegotiating the SLA.

\section{PROBLEM FORMULATION AND PRPOSED APPROACH}

\subsection{Problem formulation}

The objective is to develop a scalable signaling mechanism that provides good statistical end-to-end QoS guarantees, using very limited notification. At the same time, the proposed solution should avoid a large amount of over-provisioning of the SLAs so that higher link utilization can be achieved.

\subsection{Signaling Protocol}

The Bandwidth Broker of each domain represents the Internet Service Provider (ISP) of that domain in its business dealings with other networks. The algorithm we design is presently designed for only a single class of DiffServ traffic, though the ideas presented may be readily ex- 
tended to support multiple classes of DiffServ traffic. As in [7] we make the following assumptions:

- The ISP of a network demands money from other networks that want to reserve bandwidth for the injection of DiffServ traffic into its network.

- While the ISP for the source domain charges money from the client for sending DiffServ traffic, the sink domain does not ask money for sinking the DiffServ traffic (a kind of calling-party-pays regime).

- ISPs avoid breaking the SLAs

It is necessary for the Bandwidth Broker of the domain to dynamically renegotiate the bandwidth for DiffServ traffic with the Bandwidth Brokers of the downstream domains. This is because the amount of DiffServ traffic it needs to send fluctuates with time. For this purpose we propose a novel way to look at the dynamic re-negotiations of bandwidth for DiffServ traffic between two adjacent domains. Our algorithm is presently designed for only a single class of DiffServ traffic, though the ideas presented may be readily extended to support multiple classes of DiffServ traffic.

We model these re-negotiations as a function of available bandwidth, cost to the respective ISPs and a 'shaping probability' - the probability that the new DiffServ traffic will be shaped. The underlying assumption is that a Bandwidth Broker will update its SLA with an adjacent broker if the cost it faces for increasing the amount of reserved bandwidth is less than the revenue its ISP will get by accepting more DiffServ traffic from upstream domains and if the shaping probability is low enough. Thus we factor in the monetary transactions between the brokers into our model. The assumption that we make in the re-negotiations process also appeals intuitively since the broker will agree to upgrading the level of reserved bandwidth only if it estimates that the ISP which it represents stands to make a profit in the process.

For the purpose of renegotiating, each Bandwidth Broker (BB) maintains two metrics:

- An estimate of the expected cost it shall incur for increasing the DiffServ traffic (per unit) to each of its adjacent domains. This estimate actually evolves over time from an initial estimate as the brokers carry out dynamic re-negotiations for bandwidth. We anticipate that the expected cost shall vary over time due to the fluctuations in the way the traffic distributes. It may also vary if the brokers decide to vary their profit margins over time. An 
exponential estimator is used for the dynamic updating of the expected cost. It is anticipated that the exponential estimation shall smoothen out the variations in the expected cost. We denote $E_{j}^{X}$ as the expected cost that broker $\mathrm{X}$ maintains for its adjacent broker $j$. It represents a cost per unit of data.

- A traffic distribution matrix that specifies the probability that traffic (per unit) coming in on link $i$ will leave on link $j$. These probabilities also evolve over time from an initial estimate (the initial estimate is that the traffic is likely to go to all outgoing links with equal probability). We denote $T_{i j}^{X}$ as the (estimated) probability that traffic from broker $i$ to $\mathrm{X}$ shall be forwarded to broker $j$. Here $i$ and $j$ are brokers adjacent to $\mathrm{X}$. The idea of a traffic distribution matrix is drawn from [7].

We keep our admission control mechanism simple by admitting traffic at each domain only if the traffic being received from upstream brokers for forwarding is less than the negotiated bandwidth on the link in question and admitting the traffic shall not drive the total traffic on the link beyond negotiated levels. Of course there is a chance that DiffServ traffic even within negotiated amounts will be shaped (in some other downstream domain) since end-to-end signaling is not done. In practice more complicated admission control policies may be needed. However, for the purpose of demonstrating the efficacy of our minimal signaling inter-domain protocol, this simple protocol suffices.

\subsection{Re-negotiations process}

The notation used is:

$$
\begin{aligned}
T_{i j}^{X} & =\text { Traffic distribution probability at broker } \mathrm{X} \\
E_{j}^{X} & =\text { Expected cost at broker } \mathrm{X} \text { (for } \mathrm{j} \text { ) } \\
S_{j}^{X} & =\text { Negotiated bandwidth at } \mathrm{X} \text { (for } \mathrm{j} \text { ) } \\
D_{j}^{X} & =\text { Current Diffserv traffic from } \mathrm{X} \text { to } \mathrm{j} \\
p_{X}^{Y} & =\text { Drop probability that broker } \mathrm{Y} \text { returns to } \mathrm{X} \\
\Delta_{Y}^{X} & =\text { Change in SLA that is requested by } \mathrm{X} \text { to } \mathrm{Y} \\
Q_{X}^{Y} & =\text { Quote from } \mathrm{Y} \text { to } \mathrm{X} \\
A_{X} & =\text { Set of brokers adjacent to } \mathrm{X} \text { excluding } \mathrm{Y} \\
M_{Y}^{X} & =\text { Money that } \mathrm{X} \text { gives to } \mathrm{Y} \text { for the SLA } \\
C_{j}^{X} & =\text { Data capacity of the link from X to } \mathrm{j}
\end{aligned}
$$

The following series of steps occur during any re-negotiations process: 


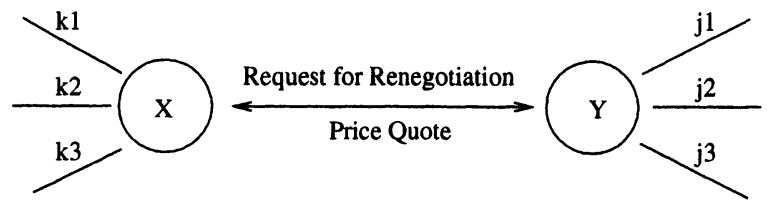

Figure 1. Re-negotiation process between brokers $\mathrm{X}$ and $\mathrm{Y}$

1 The broker for domain $\mathrm{X}\left(B B_{x}\right)$ initiates re-negotiations with the broker for domain $\mathrm{Y}\left(B B_{y}\right)$ when the quantum of DiffServ traffic between the domains $\mathrm{X}$ and $\mathrm{Y}$ exceeds some predetermined fraction $\left(f_{h}\right)$ of the currently reserved bandwidth for DiffServ traffic. By varying this fraction, the amount of over-provisioning built into the SLA can be varied.

$$
\text { if } D_{Y}^{X}>f_{h} S_{Y}^{X} \quad \Delta_{Y}^{X}=\frac{2 D_{Y}^{X}}{f_{h}+f_{l}}-S_{Y}^{X}
$$

This increment $\Delta_{Y}^{X}$ is computed so that after the negotiations, the current traffic becomes less than $f_{h} S_{Y}^{X}(n e w)$. One could use more complicated traffic estimators for computing this increment.

$2 B B_{y}$ receives a re-negotiations request from $B B_{x}$ which specifies the increase in the amount of reserved bandwidth $\mathrm{X}$ wants.

3 By using its traffic distribution matrix, $B B_{y}$ computes the expected amount of extra traffic that it $(\mathrm{Y})$ will have to pump in to each of its downstream links. Using this calculation and the expected cost of increasing its reserved bandwidth with each of its downstream domains, $B B_{y}$ then calculates the expected cost it faces for carrying the extra traffic from $\mathrm{X}$. This expected cost is sent as the price quote to $B B_{x}$. Specifically:

$$
Q_{X}^{Y}=\Delta_{Y}^{X} \sum_{j \in A_{Y}} E_{j}^{Y} T_{X j}^{Y}
$$

$4 B B_{y}$ also sends $B B_{x}$ an estimate of the probability that the new increased traffic from $\mathrm{X}$ will be shaped. $B B_{y}$ computes this probability based on the probable distribution of the traffic from $\mathrm{X}$ in to Y's downstream links and the maximum capacities of these links ( that is the maximum up to which Y can increase the DiffServ traffic on these links). Specifically the drop probability is approximated by the expression: 


$$
p_{X}^{Y}=\frac{\sum_{j \in A_{Y}} \max \left\{\left(\Delta_{Y}^{X}+S_{Y}^{X}\right) T_{X j}^{Y}-C_{j}^{Y}, 0\right\}}{\sum_{j \in A_{Y}} D_{j}^{Y}+\Delta_{Y}^{X} \sum_{j \in A_{Y}} T_{X}^{Y} j}
$$

This is the ratio of the total anticipated excess traffic on the outgoing links from $\mathrm{Y}$ to the total amount of outgoing traffic from Y.

$5 B B_{x}$ receives the quote and drop probability from $B B_{y} . B B_{x}$ updates its estimate of the cost it faces while increasing the DiffServ traffic between itself and $\mathrm{Y}$ according to the equation:

$$
E_{Y}^{X}(n e w)=\alpha E_{Y}^{X}+(1-\alpha) Q_{X}^{Y}
$$

Here $\alpha$ is a number between 0 and 1 and is a simulation parameter.

6 The re-negotiations will be successful if $B B_{x}$ sees that the drop probability is less than a predetermined (i.e. acceptable) level and the cost incurred due to the quote from $\mathrm{Y}$ is less than its estimated revenues. Let us denote estimated money that $X$ receives from its upstream brokers for pushing traffic to $\mathrm{Y}$ by $R_{Y}^{X}$. This is the sum of the revenue that $\mathrm{X}$ is already receiving and the revenue that it expects to receive from its upstream brokers. This is calculated as

$$
\begin{aligned}
R_{Y}^{X}= & \sum_{k \in A_{X}} M_{X}^{k} T_{k Y}^{X} \\
& +\sum_{k \in A_{X}} \max \left\{D_{X}^{k}-S_{X}^{k}, 0\right\} E_{X}^{k} T_{k Y}^{X}
\end{aligned}
$$

If $\mathrm{X}$ estimates its revenues to be greater than the cost incurred in pushing traffic to $\mathrm{Y}$ i.e.

$$
R_{Y}^{X}-\left(Q_{X}^{Y}+M_{Y}^{X}\right)>0
$$

it will accept the re-negotiation price sought by $\mathrm{Y}$ (provided the drop probabilities are below the predetermined level).

7 If the re-negotiations fail then $\mathrm{X}$ will again try to renegotiate with $\mathrm{Y}$ but this time with a smaller fraction of bandwidth than what was previously sought i.e $\Delta_{Y}^{X}(n e w)=\gamma \Delta_{Y}^{X}$ where $\gamma$ is a simulation parameter between 0 and 1 . This process continues until a negotiation succeeds or the additional bandwidth that is being sought becomes very small. 
$B B_{x}$ also initiates re-negotiations with $B B_{y}$ when the quantum of DiffServ traffic between the domains $\mathrm{X}$ and $\mathrm{Y}$ falls below some predetermined fraction $\left(f_{l}\right)$ of the currently allowed bandwidth for DiffServ traffic. At this point $B B_{x}$ will inform $B B_{y}$ that it wants to decrease the bandwidth for DiffServ traffic. In our model we assume that $B B_{y}$ has to accept the request of $B B_{x}$ for decreasing bandwidth. Here

$$
\Delta_{Y}^{X}=\frac{2 D_{Y}^{X}}{f_{h}+f_{l}}-S_{Y}^{X}
$$

shall be negative and

$$
M_{Y}^{X}(n e w)=M_{Y}^{X}+\Delta_{Y}^{X} E_{Y}^{X}
$$

Periodically the traffic distribution matrix at each Bandwidth Broker is updated to reflect the changing distribution patterns within the network. Specifically $B B_{x}$ will update its traffic distribution matrix using the equation:

$$
T_{i j}^{X}=\beta T_{i j}^{X}+(1-\beta) \frac{\operatorname{rate}(i, j)}{D_{X}^{i}}
$$

where $\beta$ is a simulation parameter and lies between 0 and $1, \operatorname{rate}(i, j)$ is the total traffic currently going from $i$ to $j$ through $\mathrm{X}$.

\section{EXPERIMENTAL RESULTS}

A simulator was built to study the performance of the proposed protocol. Experiments were carried out to study parameters of interest such as the expected cost and the kind of end-to-end QoS guarantees that can be provided at high network loads.

The simulations were carried out on a topology of 10 brokers. While the topology was that of a fully connected graph, different brokers were connected to different number of brokers and the capacities of the links between the brokers were chosen randomly. Further, the routing table for each broker was fixed at the start of simulations and was not varied with time. This was to prevent the routing algorithm from interfering with the performance of our signaling protocol. Each broker in our simulations is a source and sink of DiffServ traffic. We have currently implemented the simulator for only two classes of traffic namely DiffServ traffic and best effort traffic.

The objectives that we wished to achieve through the simulations were:

1 To study the total amount of DiffServ traffic (as a percentage of the total link capacities) flowing through the network over time. 


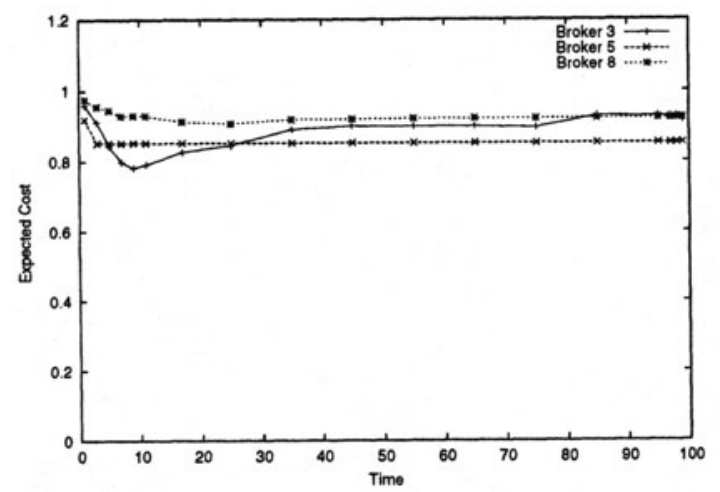

Figure 2. Evolution of Expected Cost with Time (at Broker 5).

Ideally we would want that the proposed algorithm would lead us to a situation where we will have a large amount of DiffServ traffic flowing through the network along with high QoS guarantees.

2 To study how the Quality of Service varies with the amount of over-provisioning of the SLA. Generally, we would expect that with increased over-provisioning the chances of shaping are lower and hence better QoS guarantees can be provided. Hence the yardstick on which we base our evaluation of the performance of our algorithm is whether a high QoS guarantee can be provided with sufficiently low over-provisioning.

3 To study the evolution of the metric of estimated costs that each Bandwidth Broker maintains. It is desirable that the estimated costs converge, which shall enable each Bandwidth Broker to accurately estimate the costs it faces while renegotiating for increasing the reserved bandwidth for DiffServ traffic with its adjacent domains.

\subsection{Observations and Analysis}

Figure 2 shows the evolution with time, of the expected cost at Broker 5 for its downstream brokers. This simulation was carried for $10 \%$ over provisioning (i.e. $10 \%$ of the negotiated bandwidth was kept as reserve) and it clearly shows that the expected costs settle down as time progresses. This means that as time progresses, the quote that the downstream brokers send back are very close to the expected value that the broker has for it, which is clearly very desirable. In this simulation the traffic generated at each Broker for different destinations had a fixed mean and small variance. In this case, the way traffic distributes was 


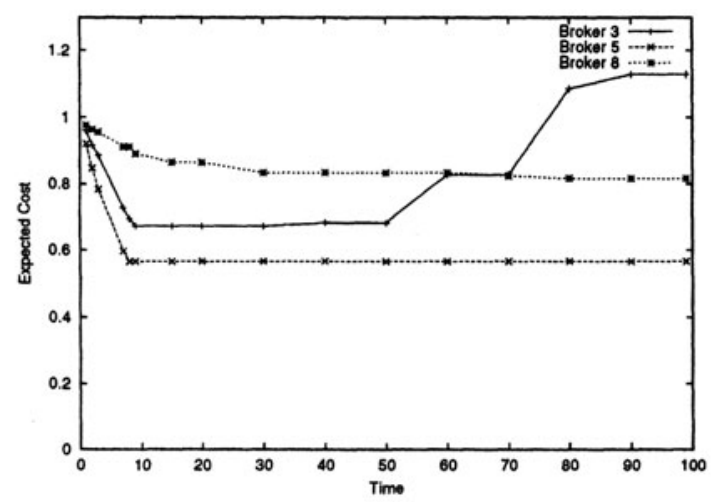

Figure 3. Evolution of Expected Cost with Time (at Broker 5). Mean traffic to various destinations is varied with time.

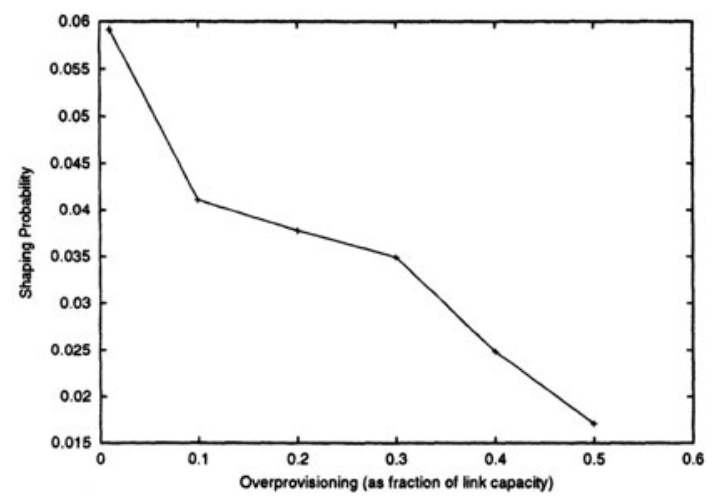

Figure 4. Shaping probability vs Over provisioning

seen to be almost constant since the routing is fixed and therefore the traffic follows the same paths. Figure 3 traces the same evolution when the traffic generated at each broker for different destinations had a time varying mean. For this case, the traffic distribution varies since the amount of traffic going to each destination changes as time progresses. This figure suggests that even though the quantum of traffic to different destinations varies with time, the expected cost is able to adapt to the changes quickly and stabilize. This can be observed from the fact that each time the traffic changes, the expected values change, but become constant after some time.

Figure 4 shows the variation of shaping probability with the amount of over provisioning. As expected, the shaping probability increases as the amount of over provisioning is reduced. However, what is encouraging is that even at very small over provisioning levels, the shaping prob- 


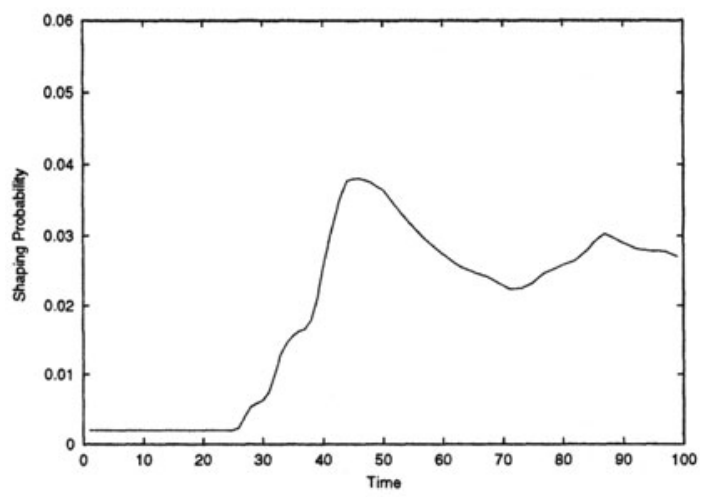

Figure 5. Evolution of Shaping probability with Time. Mean traffic to various destinations changes with time.

ability remains within acceptable limits. This implies that using our proposed protocol, the network is able to provide very high end-to-end QoS guarantees with very limited signaling. Further, it should be noted that these values for the shaping probabilities are obtained when the network is very close to saturation (as can be seen in Figure 6). This is significant since it is in the interest of the Bandwidth Brokers to be able to support high levels of traffic and still be able to provide its customers with very high QoS guarantees. Overall, therefore we can conclude that the Brokers are able to admit a large amount of traffic and still negotiate in time so that the traffic does not get dropped. Further, this also suggests that the estimates that a particular broker makes on behalf of it's downstream brokers while sending back quotes to its upstream brokers are quite accurate. Figure 5 traces the shaping probability with time when the mean traffic to different destinations changes. These simulations were carried out with $10 \%$ over provisioning. It is clear that even with variation in the traffic the system is able to do quite well and the shaping probability remains quite low.

Figure 6 provides a clue to the level of network utilization. It depicts the evolution of actual DiffServ traffic that flows as a percentage of the negotiated bandwidth with time at Broker 5. Clearly, the utilization stabilizes to an amount close to the negotiated bandwidth with an over provisioning of $10 \%$.

In all these simulations the cost to the customers is kept constant to simplify the understanding of the interaction of the quote sent by a downstream broker with the estimated inflows from upstream brokers during re-negotiations. However, it was observed that varying the costs does not affect the convergence of the algorithm and the performance of 


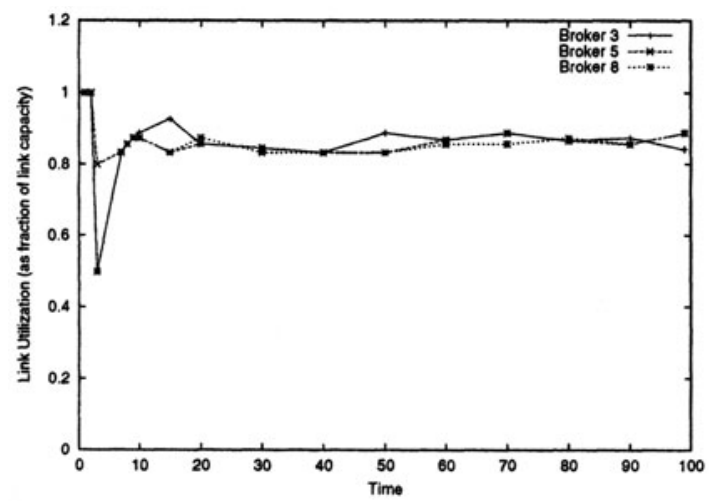

Figure 6. Evolution of Link Utilization with Time (at broker 5)

the protocol does not deteriorate in any way. Further, different topologies were used for carrying out the simulations and the performance for all the observed parameters were similar. These simulations were carried out for one hundred rounds. It was observed that carrying out more rounds of simulation did not result in any significant difference in the observations.

\section{DISCUSSION AND FUTURE WORK}

The control plane of the DiffServ architecture consists primarily of Bandwidth Brokers who establish an end-to-end quality of service through signaling between each other. The primary trade-off is between scalability and end-to-end Quality of Service. Through our simulations we were able to arrive at the following conclusions:

- Even though our signaling mechanism is minimal, in the sense that notifications and re-negotiations do not propagate beyond neighboring brokers, we are able to give sufficiently high end-to-end QoS guarantees to the clients. At the same time, the over-provisioning of the SLA is low enough to be attractive to the ISPs.

- The simulations show that the expected cost that each broker maintains converges if the variation in the traffic distribution matrix is not high. This indicates that our idea of modeling the re-negotiations not only as a function of available bandwidth but also of cost to the respective ISPs leads to a situation in which a broker shall be able to accurately estimate the costs it faces in any re-negotiations process. This is significant since the broker represents a business entity - the ISP of the domain - and will agree to any re-negotiations only when it sees a profit for itself. 
The work carried out by us demonstrates that a limited notification approach to signaling between Bandwidth Brokers is feasible as well as attractive. In the re-negotiations protocol, we have made a number of simplifying decisions for some smaller problems. These could be replaced by more carefully tailored solutions in actual implementations. For example the additional bandwidth that a broker asks for reservation to its adjacent downstream broker could be based on a better estimator of the variations in traffic. During the re-negotiation process, the downstream Bandwidth Brokers return a quote as well as a shaping probability to the upstream Bandwidth Brokers. Better estimates for the shaping probability can be developed. Lastly, it might be possible to extend the above algorithm to handle advance reservations along with immediate reservations. This will enable ISPs to give their clients greater flexibility.

\section{REFERENCES}

[1] R.Guerin, C.Partridge, and S.Shenker, "Specification of guaranteed quality of service," in RFC (Standards Track) 2212, IETF, Oct. 1997.

[2] R.Braden, L.Zhang, S.Berson, S.Herzog, and S.Jamin, "Resource reservation protocol (rsvp) - version 1 functional specification," in RFC (Standards Track) 2205, IETF, Sept. 1997.

[3] S.Blake, D.Black, M.Carlson, E.Davies, Z.Wang, and W.Weiss, "An architecture for differentiated services," in RFC (Informational) 2475, IETF, Dec. 1998.

[4] "A Discussion of Bandwidth Broker Requirements for Internet2 Qbone Deployment: Version 0.7," Available at http://www.merit.edu/working.groups/i2-qbone$\mathrm{bb} /$

[5] A.Terzis, L.Wang, J.Ogawa, and L.Zhang, "A two-tier resource management model for the internet.," in Global Telecommunications Conference, GLOBECOM, pp. 1779-1791 Vol.3, 1999.

[6] O. Schelen, A. Nilsson, J. Norrgard, and S. Pink, "Performance of QoS agents for provisioning network resources," in IWQOS, pp. 17-26, 1999.

[7] M. Gunter and T. Braun, "Evaluation of bandwidth broker signaling network protocols," in ICNP, pp. 145-152, 1999.

[8] A. Mankin, F. Baker, B. Braden, S. Bradner, M. O'Dell, A. Romanow, A. Weinrib, and L. Zhang, "Resource ReSerVation Protocol (RSVP) Version 1 Applicability Statement Some Guidelines on Deployment," in RFC (Informational) 2208, IETF, Sep. 1997. 